The Israeli Journal of Aquaculture - Bamidgeh, IJA_69.2017.1374, 11 pages

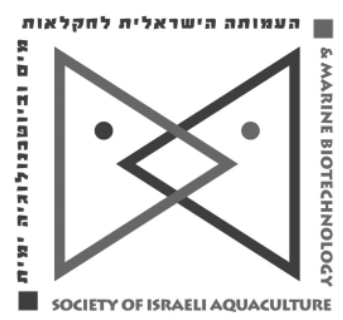

The IJA appears exclusively as a peer-reviewed on-line open-access journal at http://www.siamb.org.il. To read papers free of charge, please register online at registration form.

Sale of IJA papers is strictly forbidden.

\title{
RNA Interference Studies on the Sex- Linked Genes transformer-2and sex-lethal in the Oriental River Prawn Macrobrachium nipponense
}

\author{
Suhua Chen ${ }^{1}$, Hongtuo $\mathrm{Fu}^{1,2, *}$, Hui Qiao ${ }^{2}$, Shengming Sun ${ }^{2}$, Wenyi \\ Zhang $^{2}$, Shubo Jin ${ }^{2}$, Yongsheng Gong ${ }^{2}$, Sufei Jiang ${ }^{2}$, Yiwei Xiong ${ }^{2}$, \\ Yan $\mathbf{W} \mathbf{u}^{2}$ \\ ${ }^{1}$ Wuxi Fisheries College, Nanjing Agricultural University, Wuxi 214081, PR \\ China \\ ${ }^{2}$ Key Laboratory of Freshwater Fisheries and Germplasm Resources \\ Utilization, Ministry of Agriculture, Freshwater Fisheries Research Center, \\ Chinese Academy of Fishery Sciences, Wuxi 214081, PR China
}

Keywords: Macrobrachium nipponense; transformer-2; sex-lethal; RNA interference; sexual regulation

\begin{abstract}
The sex-lethal (sXI) and transformer-2 (tra-2) genes play key roles in the regulation of the sex differentiation pathway in insects. This study investigated the transcriptional patterns of Mntra-2 and Mnsxl in different tissues in the oriental river prawn Macrobrachium nipponense. The qPCR results showed that the highest level of Mntra-2 expression was observed in the muscle, and the highest level of Mnsxl expression was observed in the hepatopancreas. RNA interference with Mntra2/MnsxI dsRNA effectively resulted in a decrease in the expression of Mntra-2/MnsxI in the muscle, hepatopancreas and gonads in both males and females. Mntra-2 dsRNA had a significant inhibitory effect on Mnsxl mRNA expression in the testis and ovaries, but Mnsxl dsRNA did not affect Mntra-2 mRNA expression in either organ. Thus, Mntra-2 may be an upstream gene in the sex determination pathway in $M$. nipponense, and the regulatory mechanisms may be different from those observed in Drosophila melanogaster, even though these genes are homologous to the Drosophila tra-2 and $s x I$ sex-determination genes. Thus, the present results are preliminary and the sex determination pathway in $M$. nipponense needs further indepth research.
\end{abstract}

* Corresponding author. e-mail: fuht@ffrc.cn 


\section{Introduction}

The sex determination pathway in insects is well characterized in the fruit fly Drosophila melanogaster. A schematic overview of the sex determination cascade for $D$. melanogaster has been reported (Zhang et al. 2014). The master switch gene at the top of the cascade is Sex-lethal $(s x I)$, which regulates female-specific splicing of the premRNA of the transformer (tra) gene (Bell et al. 1988; Penalva and Sanchez 2003). The functional tra protein interacts with the protein product of the transformer-2(tra-2) gene, which results in the female-specific splicing of the mRNA of the bottom gene in the cascade, doublesex ( $d s x$ ) (Sievert et al. 1997). Sxl, besides regulating the femalespecific splicing of its own pre-mRNA, also regulates the female-specific splicing of the pre-mRNA of tra, resulting in the production of the functional tra protein only in females (Evans and Cline 2013). The tra-2 protein contains an RNA recognition motif domain and serine/arginine-rich regions; Moreover, it heterodimerizes with tra and binds to exonic splicing enhancer sequences present in the fourth exon (female-specific exon) of the $d s x$ pre-mRNA (Lynch and Maniatis 1996). However, genes that are homologous to the sexdetermining genes of Drosophila have not been found in all insect species (Kageyama et al. 2012; Schutt and Nothiger 2010; Heimpel et al. 2008).

In crustaceans, the molecular mechanisms regulating the expression of sex-specific and sex-determining genes have received significant attention in recent years. Several homologs of $D$. melanogaster sex-determining genes have been observed in Daphnia magna (tra and $d s x$ ), Tribolium castaneum (tra-2), Penaeus monodon (sex-related genes in the testis), Fenneropenaeus chinensis (tra-2) and Eriocheir sinensis (Dmrt and $s x /$ ) (Kato et al. 2010; Kato et al. 2011; Shukla and Palli 2013; Leelatanawit et al. 2009; Li et al. 2012; Zhang and Qiu 2010; Shen et al. 2014). Most of these genes in crustaceans exhibit high sequence similarity with their homologs in insects, but their functional consequences and sex determination pathways have not been predicted or are unclear.

The oriental river prawn Macrobrachium nipponense is an important commercial species in China. Male Macrobrachium prawns grow faster and gain more weight at harvest time than females, which makes the male prawns more economically important. Therefore, it is important to study the sex mechanism of this species, as this would help make genetic improvements and improve its production. In our previous study, we cloned two Drosophila homologous genes-tra-2 and sxl-present in M. nipponense and examined the expression patterns of these two genes (Zhang et al. 2013a, 2013b). In this study, we have further studied these two genes by analyzing their regulatory effects on each other via RNA interference (RNAi) experiments.

\section{Materials and methods}

Experimental animals and sampling. Adult healthy $M$. nipponense individuals weighing between 1.5 and $4.65 \mathrm{~g}$ (female prawns, $1.5-2.5 \mathrm{~g}$; male prawns, 3.5-4.65 g) were collected from Tai lake in Wuxi. The prawns were maintained in a re-circulating freshwater aquarium system at a constant temperature of $25^{\circ} \mathrm{C} \pm 1^{\circ} \mathrm{C}$ and fed paludina twice a day. A variety of organs, including the eyestalk, hepatopancreas, gill, heart, muscle, testis and ovary, were collected from mature male and female prawns. All tissues were dissected and washed in $1 \times$ phosphate-buffered saline $(0.01 \mathrm{M})$, frozen directly in liquid nitrogen, and stored at $-80^{\circ} \mathrm{C}$ until analysis.

RNAi experiments. RNA interference has been used successfully in our laboratory (Bai et al. 2015; Qiao et al. 2015; Li et al. 2015). dsRNA of Mntra-2 and Mnsxl were synthesized in vitro using the Transcript AidTM T7 High Yield Transcription kit (Fermentas Inc., USA) according to the manufacturer's instructions. Specific primers containing the T7 promoter site of the interfering RNA were designed using Snap Dragon (http://www.flyrnai.org/cgibin/RNAifind primers.pl). The tra-2 and sxl dsRNA synthesis primers are shown in Table 1 . The purity and integrity of dsRNA were examined by standard agarose gel electrophoresis. The concentration of dsRNA was measured at 260 $\mathrm{nm}$ with a BioPhotometer (Eppendorf, Hamburg, Germany), and then maintained at $20^{\circ} \mathrm{C}$ until use.

tra-2 dsRNA was injected into the pericardial cavity of 100 healthy mature female $M$. nipponense and 100 male $M$. nipponense individuals. The ovarian cycle of $M$. nipponense was classified into five stages based on previous results (Gao et al. 2006) and ovary 
color: Stage I, undeveloped stage characterized by oogonium proliferation in which the ovary appears transparent; Stage II, developing stage characterized by primary vitellogenesis in which the ovary appears yellow; Stage III, nearly ripe stage characterized by secondary vitellogenesis in which the ovary appears light green; Stage IV, ripe stage characterized by vitellogenesis termination and a dark green colored ovary; and Stage $V$, spent stage in which the ovary appears gray. The female prawns were selected in the proliferation stage (stage I) according to ovary color to ensure that development of the ovaries was synchronous, and they were equally distributed into two groups. In the experimental treatment group $(N=50)$, Mntra-2 dsRNA was injected into the pericardial cavity membrane of the carapace at a dose of $4 \mu \mathrm{g} / \mathrm{g}$ of body weight (BW). In the experimental control group $(\mathrm{N}=50), \mathrm{H}_{2} \mathrm{O}$ was injected at an equal dose. Male prawn of similar size were selected and also distributed equally between two treatment groups: in the experimental treatment group $(\mathrm{N}=50)$, tra-2 dsRNA was injected into the pericardial cavity membrane at a dose of $4 \mu \mathrm{g} / \mathrm{g}$, and the experimental control group $(\mathrm{N}=50)$ was injected with an equal dose of $\mathrm{H}_{2} \mathrm{O}$. Four prawns from each group were randomly collected on the 1st, 4th, 7th, 10th and 13th day after injection. The muscle, hepatopancreas and gonad were dissected, frozen immediately in liquid nitrogen, and stored at $-80^{\circ} \mathrm{C}$ until analysis.

The protocol for the $S x I$ dsRNA injection experiment was the same as described above. The hepatopancreas, muscle and gonad were collected, frozen immediately in liquid nitrogen, and stored at $-80^{\circ} \mathrm{C}$ until use.

Total RNA isolation and CDNA synthesis. Total RNA was isolated using the RNAiso Plus Reagent (TaKaRa, Japan) according to manufacturer's protocols. For all RNA samples, the A260/A280 ratio was in the range of 1.9-2.1, and RNA integrity was verified on a $1.2 \%$ agarose gel. Total RNA was treated with RNase-free DNase I (Sangon, China) to eliminate any possible genomic DNA contamination. The concentration of RNA was quantified with BioPhotometer (Eppendorf). Then, $1 \mu \mathrm{g}$ of total RNA was reverse transcribed with the iScript ${ }^{T M}$ CDNA Synthesis Kit (BIO-RAD, USA) following manufacturer's instruction.

Expression analysis by quantitative real-time $P C R$. The expression patterns of these two genes were evaluated using a quantitative real-time PCR assay on a Bio-Rad iCycler iQ5 Real-Time PCR System (Bio-Rad, USA). The qPCR amplification was carried out in a total volume of $25 \mu \mathrm{L}$ that contained $1 \mu \mathrm{L}$ of CDNA (50 ng), $10 \mu \mathrm{l}$ of SsoFast ${ }^{\mathrm{TM}}$ EvaGreen Supermix (Bio-Rad, USA), $0.5 \mu \mathrm{L}$ of $10 \mu \mathrm{M}$ specific forward and reverse primers (Table 1 ), and $13 \mu \mathrm{L}$ of nuclear water.

Table 1. Primers of sequence used

\begin{tabular}{|c|c|c|}
\hline $\begin{array}{l}\text { Primer } \\
\text { Name }\end{array}$ & Sequence $\left(5^{\prime} \rightarrow 3^{\prime}\right)$ & Description \\
\hline Tra-2-dsF & $\begin{array}{l}\text { TAATACGACTCACTATAGGGAAGCAGAGAGGA } \\
\text { CCCTAGCC }\end{array}$ & Primer for Mntra 2 dsRNA \\
\hline Tra-2-dsR & $\begin{array}{l}\text { TAATACGACTCACTATAGGGTCCTCCATATCCA } \\
\text { CTGGACC }\end{array}$ & Primer for Mntra 2 dsRNA \\
\hline sxl-dsF & $\begin{array}{l}\text { TAATACGACTCACTATAGGGCCACAGACGCTA } \\
\text { ACTGACCA }\end{array}$ & Primer for Mnsxl dsRNA \\
\hline sxl-dsR & $\begin{array}{l}\text { TAATACGACTCACTATAGGGAGTCCTGCATGA } \\
\text { TACCCAGC }\end{array}$ & Primer for Mnsxl dsRNA \\
\hline tra 2-RTF & GCCCCAGTACGTGTITAGGT & primer for qRT- PCR \\
\hline tra 2-RTR & TCCTGCGGCCATCTATTTCC & primer for qRT- PCR \\
\hline sxI-RTF & GCTGCTAAGGCAATTCTTCAGTT & primer for qRT- PCR \\
\hline SXI-RTR & CAAACCCGACTCCTCTAGGTAAC & primer for qRT- PCR \\
\hline$\beta$-actinF & TATGCACTTCCTCATGCCATC & primer for qRT- PCR \\
\hline$\beta$-actinR & AGGAGGCGGCAGTGGTCAT & primer for qRT- PCR \\
\hline
\end{tabular}

The reaction mixture was initially incubated at $95^{\circ} \mathrm{C}$ for $30 \mathrm{~s}$ to activate Hot Start Taq DNA polymerase; this was followed by 40 cycles of $95^{\circ} \mathrm{C}$ for $10 \mathrm{~s}$ and $60^{\circ} \mathrm{C}$ for $10 \mathrm{~s}$. Melting curve analysis was performed at the end of the qPCR reaction at $65-95^{\circ} \mathrm{C}$ (at $0.5^{\circ}$ increases) for $10 \mathrm{~s}$. Four replicate qPCRs were performed per sample of each four prawns, and $\beta$-actin was used as the internal control. To ensure that only one PCR 
product was amplified and detected, dissociation curve analysis of the amplification products was performed at the end of each PCR analysis. Significant differences in expression were assumed at $P$ values of $<0.05$. The relative copy number was calculated according to the $2^{-\Delta \Delta C T}$ comparative CT method (Livak and Schmittgen 2001).

Statistical analysis. Quantitative data are expressed as mean \pm SD values $(n=4)$. Statistical analysis was performed using SPSS 20.0. Statistical differences were estimated by one-way ANOVA followed by Duncan's multiple range tests. Significance was set at $P<0.05$.

\section{Results}

Tissue distribution of Mntra-2 and Mnsxl. As shown in Fig. 1, Mntra-2 mRNA was expressed in all the examined tissues of the adult prawn, with the highest mRNA levels observed in the muscles of both female and male individuals. In males, the Mntra-2 mRNA expression level is the second highest in the testis. In females, Mntra-2 mRNA expression in the ovary was lower than that in the muscle, hepatopancreas and heart.

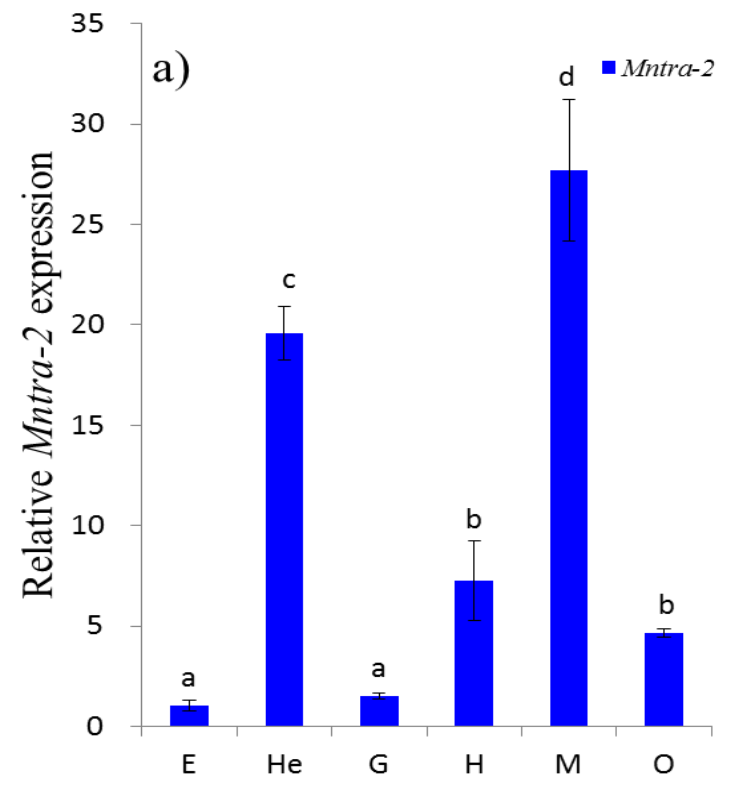

Different tissues in female prawn

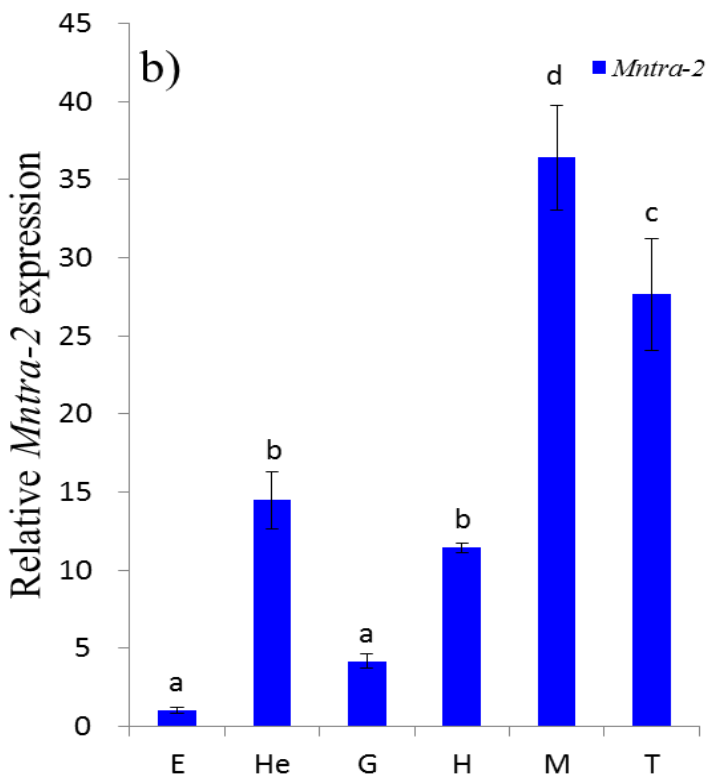

Different tissues in male prawn

Fig. 1. The expression of Mntra-2 was analyzed by quantitative real-time PCR in different tissues. The mRNA level of the two genes was normalized to the $\beta$-actin transcript level. Data are shown as the mean \pm SD values $(n=4)$. Bars marked with different letters are considered to indicate significance at $P<0.05$. E: eyestalk, He: hepatopancreas, G: gill, $H$ : heart, $M$ : muscle, O: ovary, T: testis. Statistical analyses were performed with one-way ANOVA.

Mnsxl showed the highest expression in the hepatopancreas of both female and male individuals (Fig. 2), but its expression in the gonads was lower than that in the hepatopancreas as well as the heart. 

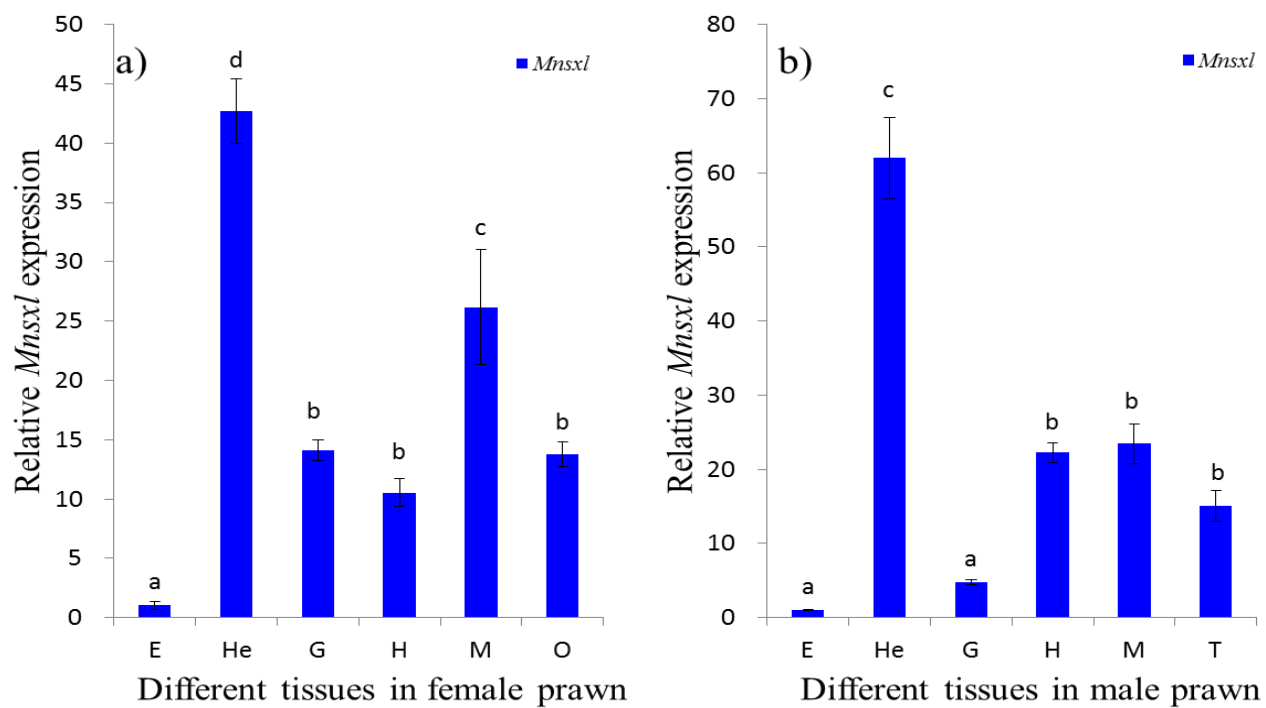

Fig. 2. The expression of $M n s x l$ in different tissues was analyzed by quantitative real-time PCR. The mRNA expression level of two genes was normalized to the $\beta$-actin transcript level. Data are shown as means \pm SD values $(n=4)$. Bars marked with different letters are considered significant at $P<0.05$. E: eyestalk, He: hepatopancreas, G: gill, $\mathrm{H}$ : heart, $\mathrm{M}$ : muscle, O: ovary, T: testis. Statistical analyses were performed with one-way ANOVA.

Effect of RNAi with Mntra-2 dsRNA on Mntra-2 expression. In the female muscle tissue (Fig. 3a), injection of Mntra-2 dsRNA resulted in downregulation of Mntra-2 expression compared with the control group. The expression of Mntra-2 began to decrease on the 1 st day and showed a $70 \%$ decrease from the 4th day to the 10th day. The expression recovered slightly on the last day. In the ovary (Fig. 3b), the mRNA level of Mntra-2 decreased and was the lowest on the 4th day, and it reached a $67.4 \%$ decrease on the 7th day.

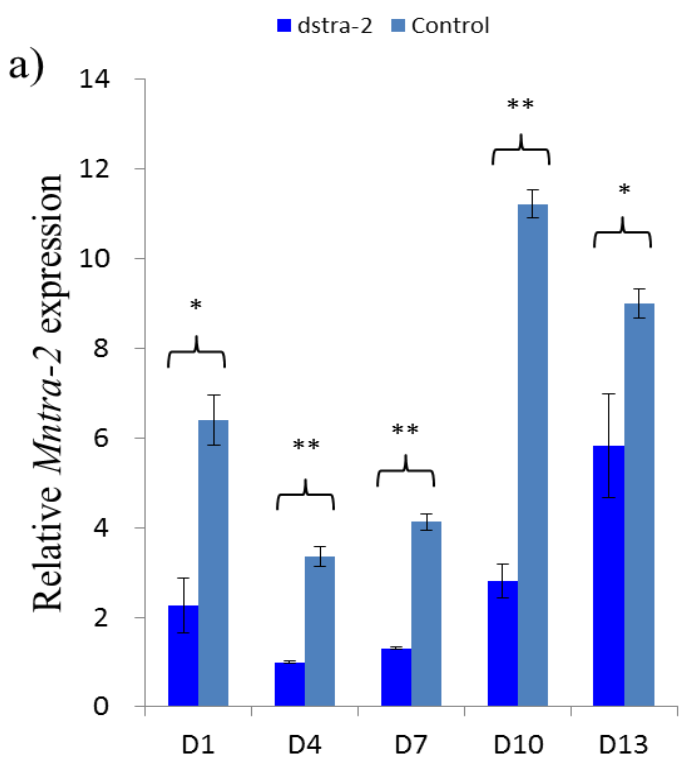

Days after Mntra-2 dsRNA

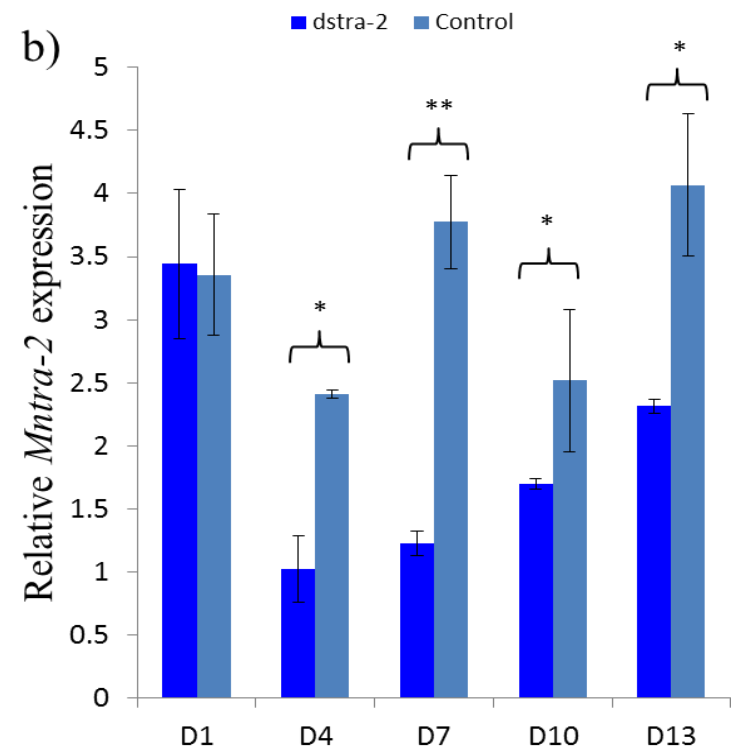

Days after Mntra-2 dsRNA

Fig. 3. Real-time PCR analysis of the effect of the tra-2 dsRNA $(4 \mu \mathrm{g} / \mathrm{g})$ injection in female prawns. (a) represents the relative Mntra-2 expression level in the muscle after RNA interference; (b) represents the relative Mntra-2 expression level in the ovary after RNA interference. The asterisk indicates significant difference between the experimental group and the control group $(* P<0.05, * * P<0.01)$ 
In males, qPCR analysis indicated that Mntra-2 dsRNA injection resulted in an $86.8 \%$ decrease in Mntra-2 expression in the muscle (Fig. 4a) and a 92\% decrease in Mntra-2 expression in the testis on the 7th day (Fig. 4b). On later experimental days, the mRNA levels increased gradually.
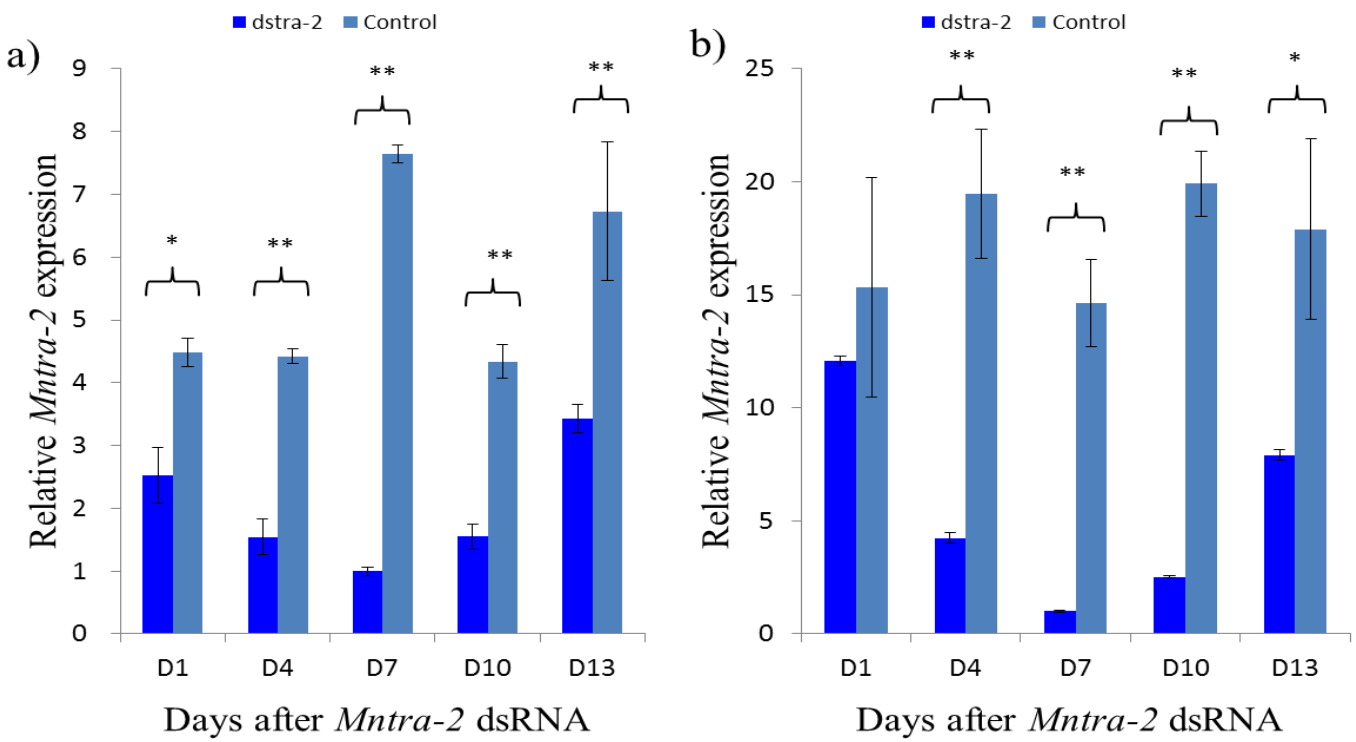

Fig.4. Real-time PCR analysis of the effect of the tra-2 dsRNA $(4 \mu \mathrm{g} / \mathrm{g})$ injection in male prawns. (a) represents the relative Mntra-2 expression level in the muscle after RNA interference; (b) represents the relative Mntra-2 expression level in the testis after RNA interference. The asterisk indicates significant difference between the experimental group and the control group $(* P<0.05, * * P<0.01)$.

Effect of RNAi with Mnsxl dsRNA on Mnsxl expression. In the hepatopancreas and ovary of Mnsxl dsRNA-injected female prawn, the expression of Mnsxl mRNA decreased by $80 \%$ and $90 \%$, respectively, in the knockdown group relative to the control group on the 4th day (Fig. $5 a$ and b).
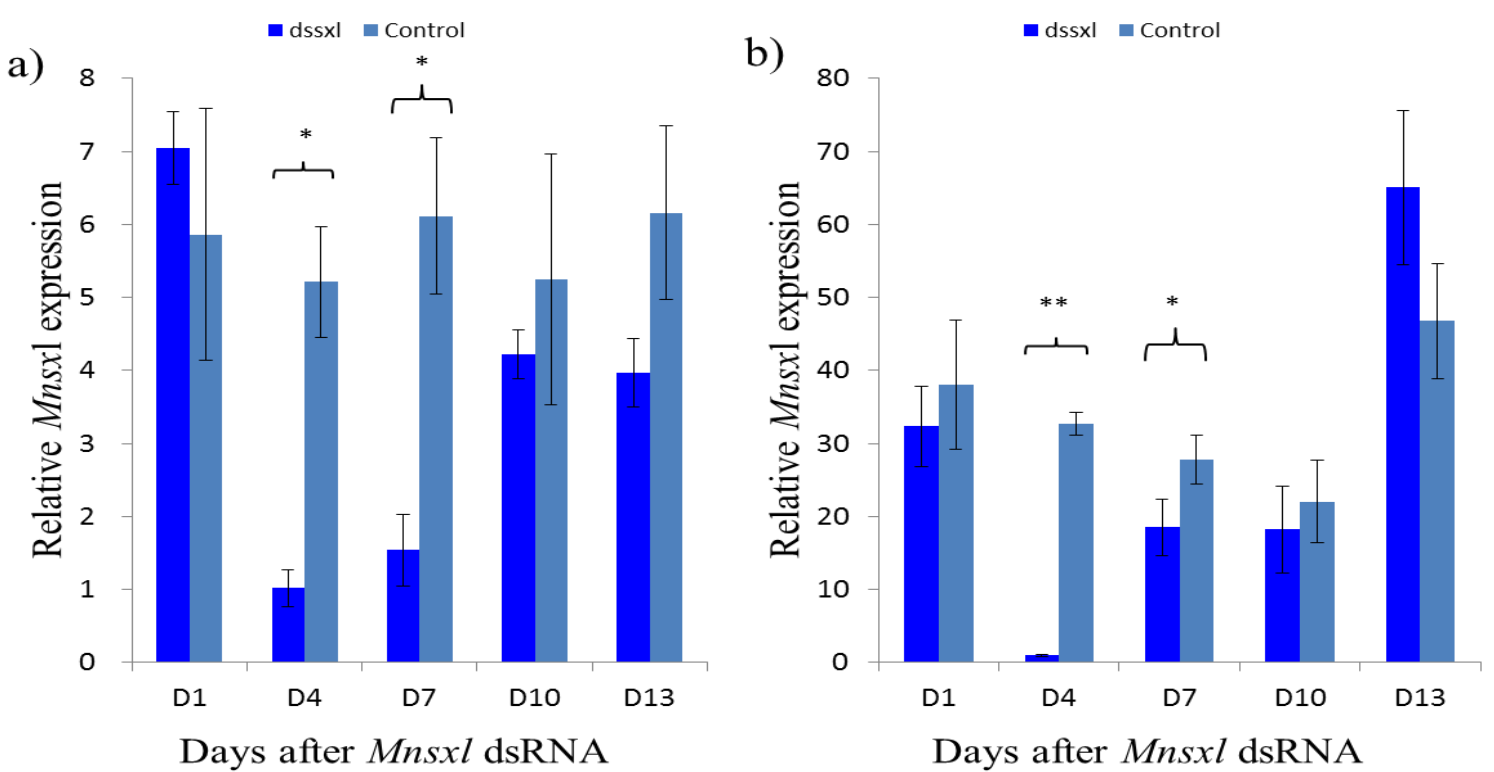

Fig. 5. Real-time PCR analysis of the effect of $s x I$ dsRNA $(4 \mu \mathrm{g} / \mathrm{g})$ injection in female prawns.

(a) represents the relative Mnsxl expression levels in the hepatopancreas after RNA inference;

(b) represents the relative Mnsxl expression levels in the ovary after RNA inference.

The asterisk indicates significant difference between the experimental group and control group $(* P<0.05, * * P<0.01)$. 
In the male hepatopancreas, the Mnsxl mRNA level reduced to $90 \%$ on the 4th day (Fig. $6 a$ ). Further, the transcript level in the testis declined by $85 \%$ on the 7th day (Fig. $6 \mathrm{~b}$ ).

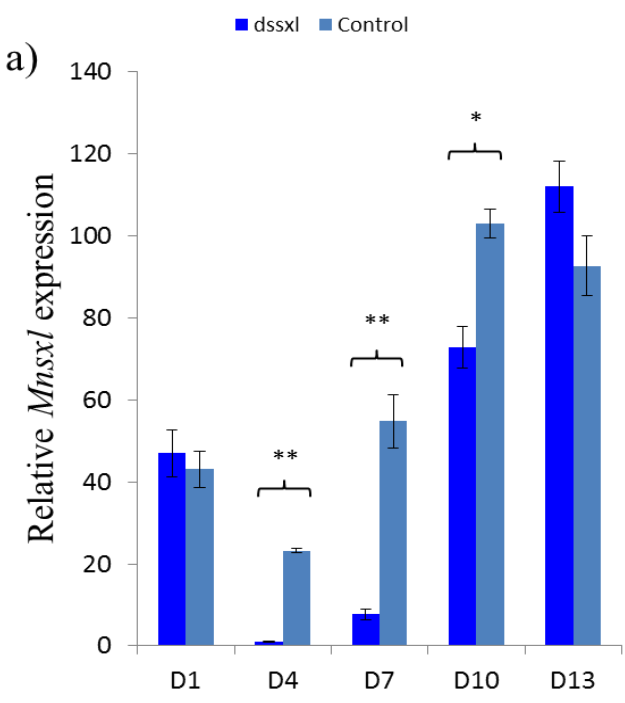

Days after Mnsxl dsRNA

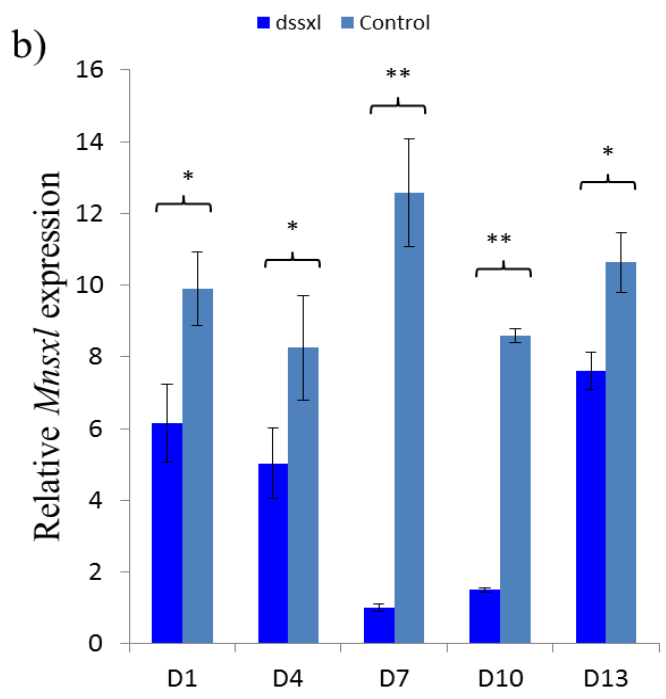

Days after Mnsxl dsRNA

Fig. 6. Real-time PCR analysis of the effect of $s x l$ dsRNA $(4 \mu \mathrm{g} / \mathrm{g})$ injection in male prawns.

(a) represents the relative Mnsxl expression level in the hepatopancreas after RNA inference;

(b) represents the relative Mnsxl expression level in testis after RNA inference.

The asterisk indicates significant difference between the experimental group and control group $(* P<0.05, * * P<0.01)$.

Influence of Mntra-2 silencing on Mnsxl gene transcription. In the Mntra-2 dsRNAinjected prawn, the expression of $M n s x l$ in the ovary reduced significantly from the 1st day to the 7th day (decreased by $70 \%$ on the 7 th day). The difference in expression compared to the control group on the 10th day and 13th day was not significant (Fig. 7a). The knockdown of Mntra-2 in the testis was successful and resulted in downregulation of the $M n s x l$ gene compared with the control animals throughout the duration of the experiment (Mnsxl expression decreased by $55 \%$ on 10 th day) (Fig. 7b).
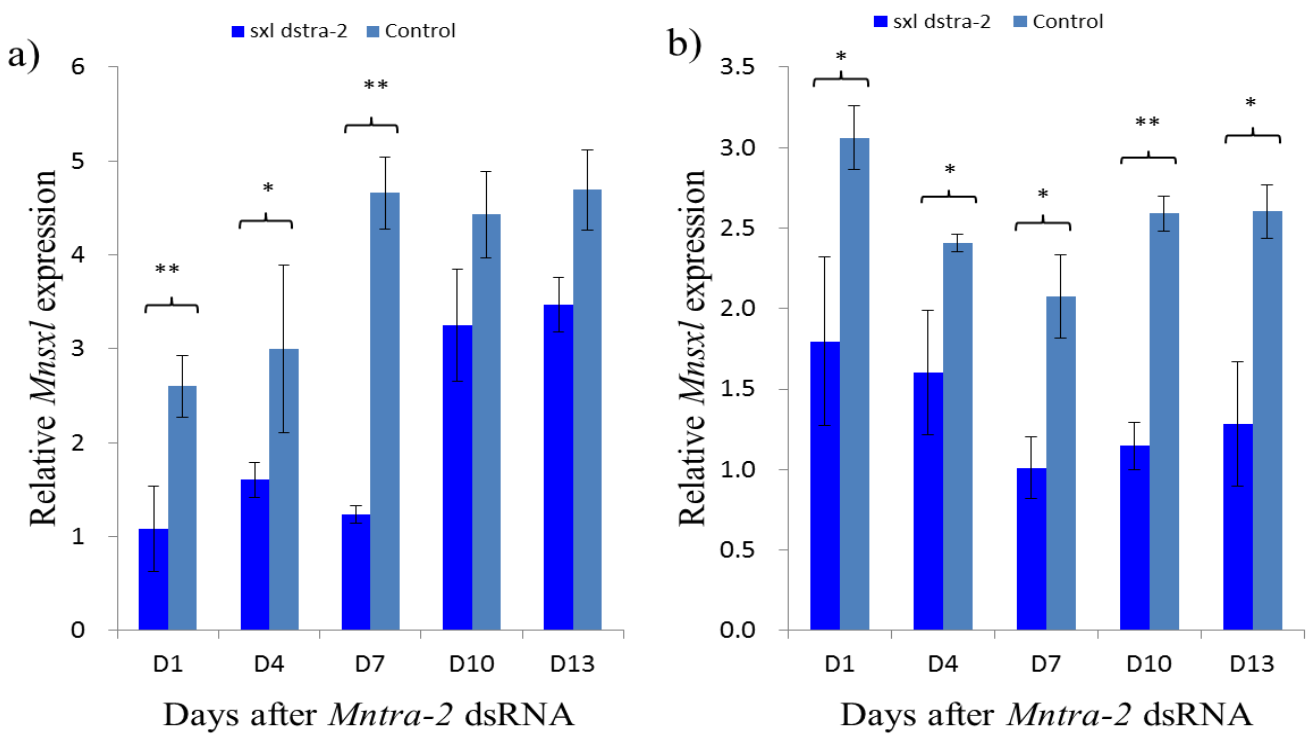

Fig. 7. Effects of Mntra-2-dsRNA-injection on Mnsxl in (a) the ovary and (b) the testis. Mnsxl mRNA levels were analyzed by GPCR. mRNA levels were normalized to $\beta$-actin mRNA levels. Data from qPCR are shown as means \pm SD values $(* P<0.05, * * P<0.01)$. 
Influence of silencing Mnsxl on Mntra-2 gene transcription. After injection of Mnsxl dsRNA, the mRNA levels of Mntra-2 in both the ovary and testis were similar in the treated and control groups (Fig. 8a and b). Although the mRNA levels in the ovary in the treated group were slightly below those in the control group, the difference in expression between these two groups was not significant $(P>0.05)$. The results confirmed that silencing of Mnsxl clearly did not affect Mntra-2 expression.

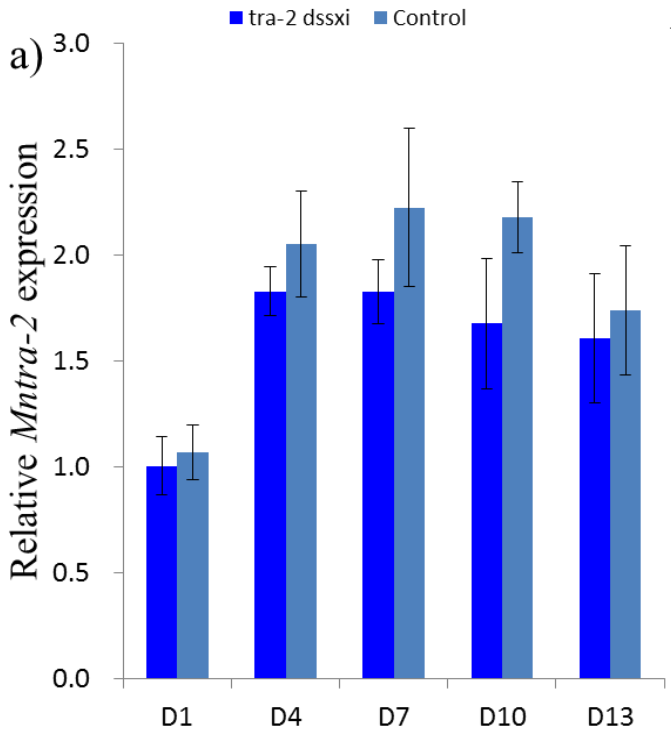

Days after Mnsxl dsRNA

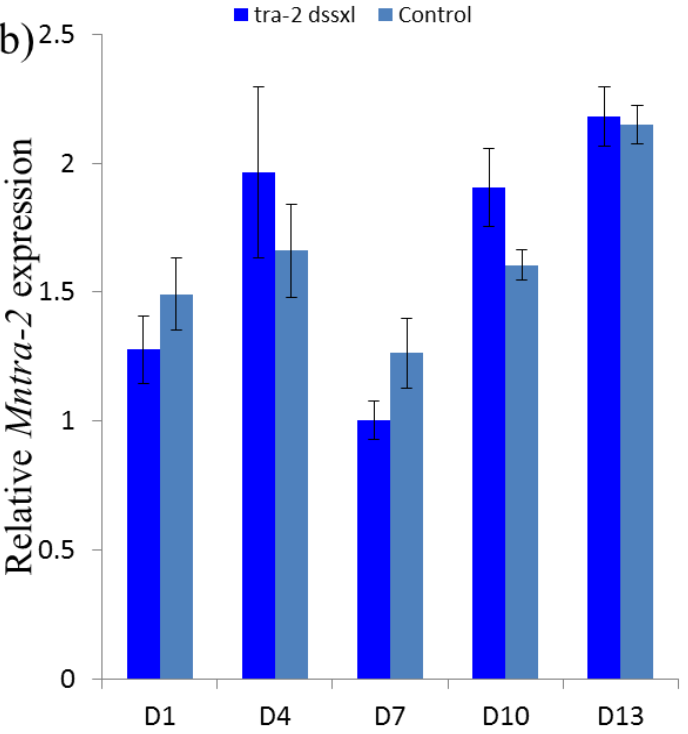

Days after Mnsxl dsRNA

Fig. 8. Effects of Mnsxl-dsRNA-injection on Mntra-2 in (a) the ovary and (b) the testis. Mntra-2 mRNA levels were analyzed by qPCR. mRNA levels were normalized to $\beta$-actin mRNA levels. Data from $\mathrm{qPCR}$ are shown as means \pm SD values $(* P<0.05, * * P<0.01)$.

\section{Discussion}

This study demonstrates, for the first time, the regulatory effects of the Mntra-2 gene on expression of the $M n s x l$ gene in $M$. nipponense. These findings lay the foundation for further studies on the genes involved in sex determination in this species, as these two genes are homologous to two genes that are involved in the sex determination cascade in $D$. melanogaster.

Mntra-2 mRNA was expressed in all examined tissues of the adult prawn, and that the mRNA levels were the highest in the muscle and second highest in the testis (Zhang, $2013 \mathrm{ba}$ ). These results were similar to those of our present study. Further, the expression level of Mntra-2 mRNA in the testis was significantly higher than that in the ovary (four times higher). The Mnsxl mRNA expression level was the highest in the hepatopancreas, followed by the muscle, but its expression in the gonads was lower than that in the hepatopancreas and muscle (Zhang et al. 2013b). In our study, too, the tissue distribution of Mnsxl was similar to that reported by Zhang et al. (2013b). The Mntra-2 mRNA level was the highest in muscle tissue, and the Mnsxl mRNA level was the highest in the hepatopancreas. Therefore, the muscle tissue and the hepatopancreas, and the gonads were used for the remaining experiments.

In previous RNAi studies, we have shown that $4 \mu \mathrm{g}$ of dsRNA per gram of shrimp is effective to suppress the expression of several genes (Bai et al. 2015; Qiao et al. 2015). In this study, injection of dsRNA successfully reduced the transcriptional levels of the target genes (Mntra-2 and Mnsxl) in the examined tissues at a dose of $4 \mu \mathrm{g}$ per gram BW. In particular, Mntra-2 dsRNA inhibited the Mntra-2 mRNA level in the testis more effectively: the expression of Mntra-2 in the testis in the control group was more than 15 times that in the treatment group on the 7th day. Moreover, the expression of Mnsxl in the ovary in the control group was more 30 times that in the experimental group on the 4th day. In $D$. melanogaster, tra-2 is a key gene that acts on the cascades regulating both somatic sexual differentiation and male fertility (Belote and Baker 1983). sxl functions as the master switch gene for sex determination in $D$. melanogaster (Bell et al. 1988; Zhang et al. 2014; Gempe and Beye 2011). Based on these reports, we believe 
that these two genes (Mntra-2 and $M n s x l$ ) may be related to sex determination in $M$. nipponense.

The MnsxI mRNA levels in the testis and ovary were significantly reduced by injection of Mntra-2 dsRNA, but the Mntra-2 mRNA levels were not reduced by injection of MnsxI dsRNA. These results indicate that Mntra-2 has a positive regulatory effect on $M n s x I$ at the mRNA level in the gonads, which means that Mntra-2 may be an upstream gene in the sex determination pathway in $M$. nipponense. This is different from the relationship between $s x l$ and tra-2 in D. melanogaster (Zhang et al. 2014). In Bombyx mori, too, the chromosomal sex determination mechanism is distinct from that in $D$. melanogaster; moreover, female sex in $B$. mori is determined by the presence of a dominant feminizing factor (fem) and not $s x l$, which plays a sex-independent role in $B$. mori (Niimi et al. 2006; Suzuki 2010). A tra homolog has not been found in the Bombyx genome, but Bmtra-2 is reportedly required for normal testis development (Suzuki et al. 2012; Sakai et al. 2014). Thus, genes that are homologous to the Drosophila sex-determining genes may have different functions in other insect species and should therefore be investigated in depth in other species too.

There is still much research lacking on sex determination in crustaceans. For example, in the freshwater prawn species Macrobrachium rosenbergii and $F$. chinensis, in which sex is determined chromosomally, with the females being heterogamous (ZW) and the males being homogamous ( $Z Z)$, the sex determination pathway is currently unclear (Malecha et al. 1992; Li et al. 2003). Further, the genotype of M. nipponense has not been determined, and several sex-specific and sex-determining genes have not been found or researched in depth. This study is still in the preliminary stage of exploring the sex regulatory mechanism of $M$. nipponense.

\section{Conclusion}

We examined the tissue distribution of Mntra-2 and Mnsxl in M. nipponense. Injection of dsRNA resulted in a successful decrease in the transcriptional levels of the target genes. The RNAi results along with other evidence from the literature indicate that Mntra-2 and Mnsxl play important roles in sex differentiation in M. nipponense. However, sex regulation of these two genes was different from that in $D$. melanogaster. Our findings indicate that Mntra-2 may be an upstream gene involved in the expression of $M n s x l$ in $M$. nipponense, but further research is required to completely understand the sex-determination pathway in this species.

\section{Acknowledgements}

This work was supported by the National Natural Science Foundation of China [grant number 31572617]; the Freshwater Fisheries Research Center, China Central Governmental Research Institutional Basic Special Research Project from the Public Welfare Fund [grant number 2015JBFM05]; the Fund of Independent Innovation of Agricultural Sciences of Jiangsu province [grant number CX (15)1012-4]; the Three Aquatic Projects of Jiangsu Province [grant number D2015-16]; and the Science and Technology Development Fund of Wuxi [grant number CLE02N1514].

\section{References}

Bai, H.K., Qiao, H., Li, F.J., Fu, H.T., Sun, S.M., Zhang, W.Y., Jin, S.B., Gong, Y.S., Jiang, S.F., and Y.W. Xiong, 2015. Molecular characterization and developmental expression of vitellogenin in the oriental river prawn Macrobrachium nipponense and the effects of RNA interference and eyestalk ablation on ovarian maturation. Gene, 562: 2231.

Baker, B.S., and J.M. Belote, 1983. Sex determination and dosage compensation in Drosophila melanogaster. Annu. Rev. Genet., 17: 345-393.

Bell, L.R., Maine, E.M., Schedl, P., and T.W. Cline, 1988. Sex-lethal, a Drosophila sex determination switch gene, exhibits sex-specific RNA splicing and sequence similarity to RNA binding proteins. Cell, 55: 1037-1046. 
Evans, D.S. and T.W. Cline, 2013. Drosophila switch gene Sex-lethal can bypass its switch-gene target transformer to regulate aspects of female behavior. Proc. Natl. Acad. Sci., 110: e4474-e4481.

Gao, X.G., Liu, H., Xu, J.N., and S.L. Cai, 2006. Study on Site of Vitellogenin Synthesis in the Freshwater Prawn Macrobrachium Nipponese. Biotechnology Bulletin, supplement 438-444. (In Chinese)

Gempe, T. and M. Beye, 2011. Function and evolution of sex determination mechanisms, genes and pathways in insects. Bioessays, 33: 52-60.

Gempe, T., Hasselmann, M., Schiøtt, M., Hause, G., Otte, M., and M. Beye, 2009. Sex determination in honeybees: two separate mechanisms induce and maintain the female pathway. Plos Biol., 7: e1000222.

Heimpel, G.E. and J.G. de Boer, 2008. Sex determination in the Hymenoptera. Annu. Rev. Entomol., 53: 209-230.

Kageyama, D., Narita, S., and M. Watanabe, 2012. Insect sex determination manipulated by their endosymbionts: incidences, mechanisms and implications. Insects, 3: 161-199.

Kato, Y., Kobayashi, K., Oda, S., Tatarazako, N., Watanabe, H., and T. Iguchi, 2010. Sequence divergence and expression of a transformer gene in the branchiopod crustacean, Daphnia magna. Genomics, 95: 160-165.

Kato, Y., Kobayashi, K., Watanabe, H., and T. Iguchi, 2011. Environmental sex determination in the branchiopod crustacean Daphnia magna: deep conservation of a Doublesex gene in the sex-determining pathway. Plos Genet., 7: e1001345.

Leelatanawit, R., Sittikankeaw, K., and P. Yocawibun, 2009. Identification, characterization and expression of sex-related genes in testes of the giant tiger shrimp Penaeus monodon. Comp. Biochem. Phys. A, 152: 66-76.

Li, F.J., Bai, H.K., Xiong, Y.W., Fu, H.T., Jiang, S.F., Jiang, F.W., Jin, S.B., Sun, S.M., Qiao, H., and W.Y. Zhang, 2015. Molecular characterization of insulin-like androgenic gland hormone-binding protein gene from the oriental river prawn Macrobrachium nipponense and investigation of its transcriptional relationship with the insulin-like androgenic gland hormone gene. Gen. comp. endocrinology, 216: 152-160.

Li, F., Xiang, J., Zhang, X., Zhou, L., Zhang, C., and C. Wu, 2003. Gonad development characteristics and sex ratio in triploid Chinese shrimp (Fenneropenaeus chinensis). Mar. Biotechnol., 5: 528-535.

Li, S., Li, F., Wen, R., and J. Xiang, 2012. Identification and characterization of the sex-determiner transformer-2 homologue in Chinese shrimp, Fenneropenaeus chinensis. Sex. Dev., 6: 267-278.

Livak, K.J. and T.D. Schmittgen, 2001. Analysis of relative gene expression data using real-time quantitative PCR and the Method. Methods, 25: 402-408.

Lynch, K.W. and T.Maniatis, 1996. Assembly of specific SR protein complexes on distinct regulatory elements of the Drosophila doublesex splicing enhancer. Genes dev., 10: 2089-2101.

Malecha, S.R., Nevin, P.A., Ha, P., Barck, L.E, Lamadrid-Rose, Y., Masuno, S., and D. Hedgecock, 1992. Sex-ratios and sex-determination in progeny from crosses of surgically sex-reversed freshwater prawns, Macrobrachium rosenbergii. Aquaculture, 105: 201-218.

Niimi, T., Sahara, K., Oshima, H., Yasukochi, Y., Ikeo, K., and W. Traut, 2006. Molecular cloning and chromosomal localization of the Bombyx Sex-lethal gene. Genome, 49: 263-268.

Penalva, L. O. and Sanchez, L. 2003. RNA binding protein Sex-lethal (Sxl) and control of Drosophila sex determination and dosage compensation. Microbiol. Mol. Biol. Rev., 67: 343-359.

Qiao, H., Xiong, Y.W., Zhang, W.Y., Fu, H.T., Jiang, S.F., Sun, S.M., Bai, H.K., Jin, S.B., and Y.S. Gong, 2015. Characterization, expression, and function analysis of gonad-inhibiting hormone in Oriental River prawn, Macrobrachium nipponense and its induced expression by temperature. Comp. Biochem. Phys. A, 185: 1-8.

Sakai, H., Aoki, F., and M.G. Suzuki, 2014. Identification of the key stages for sex determination in the silkworm, Bombyx mori. Dev. Genes. Evol., 224: 119-123. 
Schutt, C. and R. Nothiger, 2000. Structure, function and evolution of sex-determining systems in Dipteran insects. Development, 127: 667-677.

Shen, H., Hu, Y., and X. Zhou, 2014. Sex-lethal gene of the Chinese mitten crab Eriocheir sinensis: cDNA cloning, induction by eyestalk ablation, and expression of two splice variants in males and females. Dev. Genes. evol., 224: 97-105.

Shukla, J.N. and S.R. Palli, 2013. Tribolium castaneum Transformer-2 regulates sex determination and development in both males and females. Insect biochem. Molec., 43: 1125-1132.

Sievert, V., Kuhn, S., and W. Traut, 1997. Expression of the sex determining cascade genes Sex-lethal and doublesex in the phorid fly Megaselia scalaris. Genome, 40: 211214.

Suzuki, M.G., Imanishi, S., Dohmae, N., Asanuma, M., and S. Matsumoto, 2010. Identification of a male-specific RNA binding protein that regulates sex-specific splicing of Bmdsx by increasing RNA binding activity of BmPSI. Mol. Cell. Boil., 30: 5776-5786.

Suzuki, M.G., Suzuki, K., Aoki, F., and M. Ajimura, 2012. Effect of RNAi-mediated knockdown of the Bombyx mori transformer-2gene on the sex-specific splicing of Bmdsx pre-mRNA. Int. J. Dev. Biol., 56: 693-699.

Zhang, E.F. and G.F. Qiu, 2010. A novel Dmrt gene is specifically expressed in the testis of Chinese mitten crab, Eriocheir sinensis. Dev. genes evol., 220: 151-159.

Zhang, Y.P., Fu, H.T., Qiao, H., Jin, S.B., Jiang, S.F., Xiong, Y.W., and X. Zhang, 2013a. Molecular Cloning and Expression Analysis of Transformer-2 Gene During Development in Macrobrachium nipponense (de Haan 1849). Journal of the world Aquaculture Society 44: 338-349.

Zhang, Z., Klein, J., and Nei, M. 2014. Evolution of the Sex-lethal gene in insects and origin of the Sex-determination system in Drosophila. J. world. Aquacult. Soc., 78: 5065.

Zhang, Y.P., Qiao, H., Zhang, W.Y., Sun, S.M., Jiang, S.F., Gong, Y.S., and H.T. Fu, 2013b. Molecular cloning and expression analysis of two sex-lethal homolog genes during development in the oriental river prawn, Macrobrachium nipponense. Genet. Mol. Res., 12: $4698-4711$. 